\title{
The 4.2-m William Herschel Telescope as ELT testbed facility
}

\author{
René G. M. Rutten ${ }^{1}$, Richard M. Myers ${ }^{2}$ and Tim J. Morris ${ }^{2}$ \\ ${ }^{1}$ Isaac Newton Group of Telescopes, Apartado de Correos 321, \\ E-38700 Santa Cruz de la Palma, Spain, email: rgmr@ing.iac.es \\ ${ }^{2}$ Department of Physics, University of Durham, Rochester Building, Science Laboratories, \\ South Road, Durham DH1 3LE, UK
}

\begin{abstract}
The technical developments required to build the future Extremely Large Telescopes will be very demanding. Some of these developments, for instance in the field of Adaptive Optics, will rely on experimental work to test new techniques and concepts. The 4.2-m William Herschel Telescope located at a representative high-quality observing site, and with its stable Nasmyth optical bench for general access and its common-user Rayleigh laser beacon, is well placed to play a role as a testbed facility for such activities.
\end{abstract}

Keywords. instrumentation: adaptive optics; instrumentation: high angular resolution

For future Extremely Large Telescopes (ELTs) to deliver the desired science potential many important technical problems need to be resolved. Problems related to the telescope structure, instruments, and the use of Adaptive Optics technology. Examples are: phasing of the primary mirror segments under demanding environmental conditions, complications due to axial aberrations of finite-focus laser guide stars, a depth-of-field that is smaller than the Sodium layer thickness, huge laser beacon spot elongation across the pupil, and the general complexity of having to operate many lasers to overcome the cone effect.

In order to resolve these technical challenges with confidence, experimental work under realistic conditions will be required. Such experiments demand a sizeable telescope with appropriate facilities and good observing conditions to carry out these tests. The $4.2-\mathrm{m}$ William Herschel Telescope (WHT) at the Roque de los Muchachos Observatory on the island of La Palma, Spain, is well placed to play a significant role as testbed platform and is already being employed for this.

Experiments that are currently being carried out include tests of a novel laser beacon wavefront sensing concept to overcome the severe problem of perspective elongation (Kellner et al. 2004), and new, efficient optical methods for phasing of primary mirror segments using a pyramid wavefront sensor (Esposito et al. 2005). Planned experiments include new methods to avoid the severe problem facing ELTs of focal anisoplanatism, by sensing wavefront distortions directly in the Earth's atmosphere using the upward path of a laser beacon (Bonaccini et al. 2004).

Realistic ELT laser beacon experiments can be conducted by scaling the telescope pupil to the size of existing telescope apertures. For example, a 50m ELT with a Sodium laser beacon may be modelled by scaling down to a $4.2-\mathrm{m}$ telescope with a Rayleigh laser projected at $8 \mathrm{~km}$ altitude.

ELT developments will profit from realistic experiments that require a reasonably big telescope, a representative observing site, a powerful Rayleigh laser with laser launch 


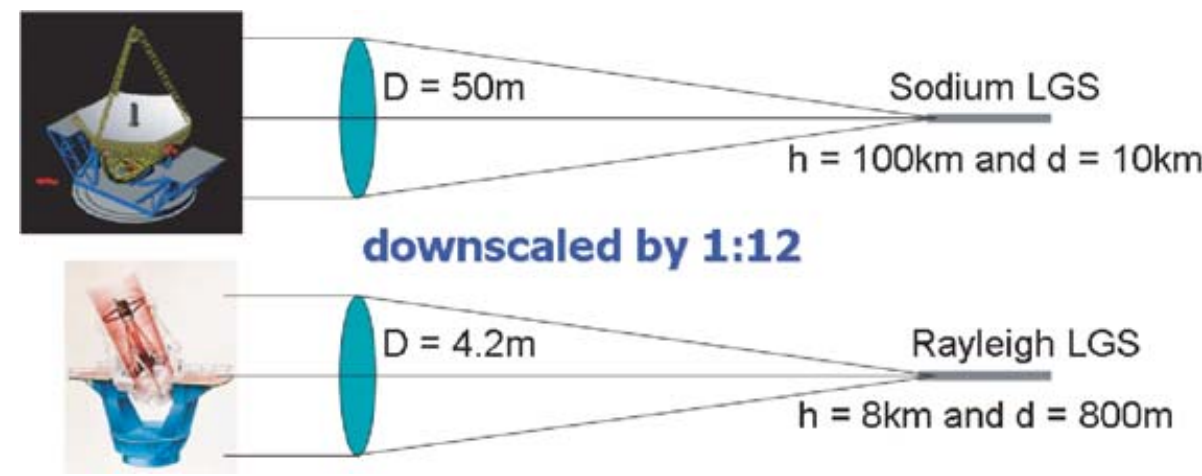

Figure 1. Scaling of a Sodium laser beacon for the ELT geometry to a 4-m telescope with a Rayleigh laser beacon allows realistic ELT experiments to be conducted.

infrastructure, a stable instrument platform and possibly an AO system. All of this is available at the WHT.

The WHT possesses a Nasmyth focus laboratory for experimental activities. A $30 \mathrm{~W}$ pulsed $515 \mathrm{~nm}$ laser and launch system is being developed for artificial beacon Adaptive Optics, and this system will also be available for experimental work, as a secondary goal.

The laser and beam launch system will be set up to produce a beacon at a distance of $20 \mathrm{~km}$, but by tuning the shutter delay time and focus, the beacon can also be used at very different elevations. By changing the shutter range gate the physical beacon length can be tuned as well. Finally, the laser power can be reduced if necessary, allowing a wide range of parameters to be explored in experiments, such as for instance to gauge the true performance of ground layer adaptive optics.

The WHT also possesses a suite of meteorological and atmospheric monitoring tools. Continuous atmospheric turbulence profiling using a Multi Aperture Scintillation Sensor (MASS, Tokovinin et al. 2003) is expected to become available from 2006 onwards.

In summary, the advantages of the WHT, its infrastructure, and the observing site provide a unique facility for ELT-related experimental research.

\section{References}

Bonaccini, C., Myers, R.M., Zappa, F., Love, G.D., Morris, T.J., Hackenberg, W.K.P., Wilson, R.W. \& Buscher, D.F. 2004, SPIE 5490, 1315

Esposito, S., Pinna, E., Puglisi, A., Tozzi, A. \& Stefanini, P. 2005, Optics Letters 30 (19), 2572 Kellner, S., Ragazzoni, R., Gassler, W., Diolaiti, E., Morris, T.J., Saunter, C.D., Myers, R., Farinato, J., Arcidiacono, C. \& Ghedina, A. 2004, SPIE 5490, 989

Tokovinin, A., Kornilov, V., Shatsky, N. \& Voziakova, O. 2003, MNRAS 343, 891 